University of Nebraska - Lincoln

DigitalCommons@University of Nebraska - Lincoln

Roger Kirby Publications

Research Papers in Physics and Astronomy

May 1993

\title{
Magnetization reversal and defects in Co/Pt multilayers
}

J.X. Shen

University of Nebraska - Lincoln

Roger D. Kirby

University of Nebraska-Lincoln, rkirby1@unl.edu

K. Wierman

University of Nebraska - Lincoln

Z.-S. Shan

University of Nebraska - Lincoln

David J. Sellmyer

University of Nebraska-Lincoln, dsellmyer@unl.edu

See next page for additional authors

Follow this and additional works at: https://digitalcommons.unl.edu/physics_kirby

Part of the Physics Commons

Shen, J.X.; Kirby, Roger D.; Wierman, K.; Shan, Z.-S.; Sellmyer, David J.; and Suzuki, T., "Magnetization reversal and defects in Co/Pt multilayers" (1993). Roger Kirby Publications. 35.

https://digitalcommons.unl.edu/physics_kirby/35

This Article is brought to you for free and open access by the Research Papers in Physics and Astronomy at DigitalCommons@University of Nebraska - Lincoln. It has been accepted for inclusion in Roger Kirby Publications by an authorized administrator of DigitalCommons@University of Nebraska - Lincoln. 


\section{Authors}

J.X. Shen, Roger D. Kirby, K. Wierman, Z.-S. Shan, David J. Sellmyer, and T. Suzuki 


\title{
Magnetization reversal and defects in Co/Pt multilayers
}

\author{
J. X. Shen, R. D. Kirby, K. Wierman, Z. S. Shan, and D. J. Sellmyer \\ Behlen Laboratory of Physics and Center for Materials Research and Analysis. \\ University of Nebraska, Lincoln, Nebraska 68588-0111
}

T. Suzuki

IBM Almaden Research Center, 650 Harry Road, San Jose, California 95120-6099

(Co $3 \AA /$ Pt $10 \AA) \times N(N=8,16$, and 30 bilayers) multilayer thin films were prepared by sputtering onto an 850 -Â-thick SiN layer that had been deposited on a silicon (111) substrate. We used the polar Kerr effect to measure the time dependence of magnetization reversal over the temperature range 90-300 K. Direct domain observations were also carried out. The results show that the domain expansion process depends strongly on the number of bilayers. Uniform domain expansion was found only in the thinner samples. From the time dependence of the magnetization reversal measurements over the temperature range $90-300 \mathrm{~K}$, the activation energy and volume associated with domain wall motion were found to be $1.2 \mathrm{eV}$ and $2.3 \times 10^{-18}$ $\mathrm{cm}^{3}$ for $N=8$ sample. The $N=16$ and $N=30$ samples seem to have a broad distribution of activation energies. Our observations suggest that both the coercivity and magnetization reversal are controlled by the defects that come from the interface between the Co and Pt.

\section{INTRODUCTION}

$\mathrm{Co} / \mathrm{Pt}$ multilayers are of considerable practical interest because of their potential applications as magneto-optical recording media. In particular, Co $3 \AA /$ Pt $10 \AA$ multilayers have the necessary large perpendicular magnetic anisotropy and large Kerr rotation in the blue for them to be considered as strong candidates for the next generation of optical recording media. ${ }^{1}$ Magnetic thin films with large perpendicular anisotropy usually display a strong intrinsic magnetic aftereffect due to thermal activation. This effect is very sensitive to the values of the magnetic parameters such as the magnetic anisotropy constant $K_{U}$ and the saturation magnetization $\boldsymbol{M}_{S}$, and to structural defects either on the micro- or nanoscale. ${ }^{2-4}$ In this article, we report a study of magnetization reversal in (Co $3 \AA$ A $/ \mathrm{Pt} 10 \AA) \times N$ multilayer samples over the temperature range $90-300 \mathrm{~K}$. These measurements, when combined with direct domain observations by polarized light microscopy, permit us to determine in some detail how defects influence magnetization reversal and coercivity in these materials.

\section{EXPERIMENT}

(Co $3 \AA / \mathrm{Pt} 10 \AA) \times N, N=8,16$, and 30 , multilayer thin films were prepared by if (for $\mathrm{Co}$ ) and dc (for Pt) magnetron sputtering. The substrate is silicon (111). Between the substrate and the Co/Pt there is a 850 - $\AA$ thick $\mathrm{SiN}$ buffer layer. Polarized light microscopy was used to observe the domain structure. Polar Kerr rotation measurements were carried out in the temperature range from $90 \mathrm{~K}$ to room temperature with light of wavelength $6328 \AA$ using apparatus described in Ref. 5 . The procedure to measure time dependence of magnetization is as follows: The films were first magnetically saturated using a field of 15 $\mathrm{kOe}$ (greater than $5 H_{C}$ in every case) perpendicular to the film plane. The field is believed to be large enough to establish a saturated state, as the Kerr rotation is constant between 5 and $15 \mathrm{kOe}$. Once the sample was saturated, a reversed field very close to $H_{C}$ was applied, and the Kerr rotation was monitored as a function of time.

\section{RESULTS AND DISCUSSION}

Figure 1 shows the polar Kerr rotation hysteresis loops of the Co $3 \AA / \mathrm{Pt} 10 \AA$ multilayers. The transitions from the $+M_{S}$ state to the $-M_{S}$ state are very sharp for all three samples, but the $N=30$ sample shows a relatively slow approach to saturation. This is apparently due to nonuniform domain wall expansion, as will be discussed in detail later.

Figure 2 shows the polar Kerr rotation as a function of time after a reversed magnetic field is applied for the three samples, both at 96 and $300 \mathrm{~K}$. The six curves in each figure correspond to different values of the reversing field, with the uppermost curve corresponding to the smallest

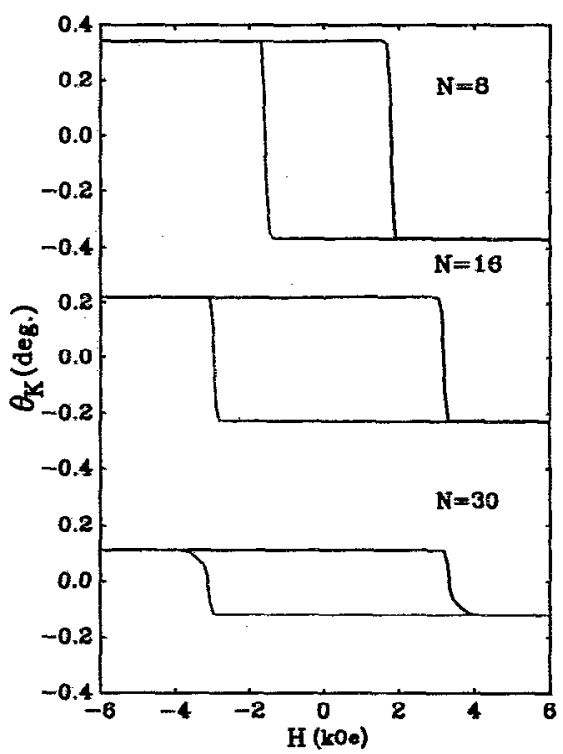

FIG. 1. Kerr rotation hysteresis loops for $(\mathrm{Co} 3 \AA / \mathrm{Pt} 10 \AA) \times N$. 


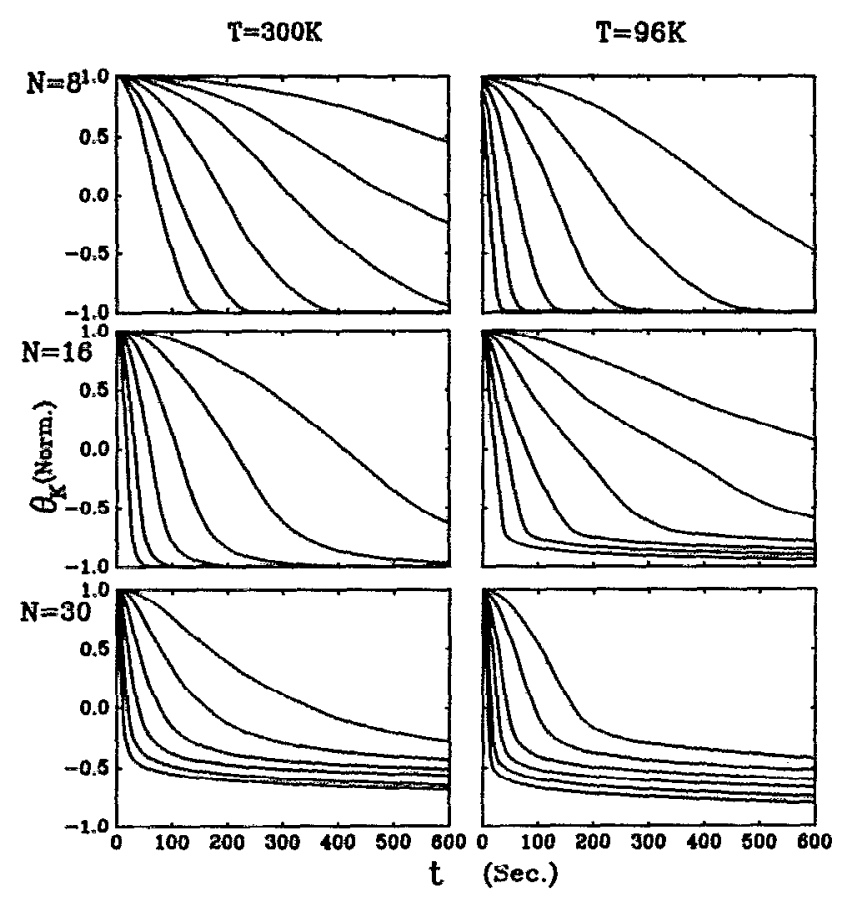

FIG. 2. Kerr rotation as a function of time after magnetization reversal for (Co $3 \AA / \mathrm{Pt} 10 \AA) \times N$ for several different values of the reversing field at $T=300 \mathrm{~K}$ and $T=96 \mathrm{~K}$. Different curves correspond to the different reversing flelds, with the uppermost curve corresponding to the smallest field.

field. The basic shapes for all of the curves are similar: There is an initially small decay rate, followed by a much more rapid decay rate and a slow approach to approximately complete reversal. The general shape of the curves suggests that the initial decay rate is limited by a slow nucleation processes, but once nucleated the domains grow quite rapidly. ${ }^{2,4}$

The reversal behavior at long times is quite different for the three samples. For the $N=8$ and $N=16$ samples at room temperature, the magnetization reverses completely even for reversing fields much smaller than the coercivity. But for the $N=30$ sample at room temperature and at 96 $\mathrm{K}$ and the $N=16$ sample at $96 \mathrm{~K}$, complete magnetization reversal can be achieved only if the reversing field is larger than the coercivity. For fields less than $H_{C}$ in these cases, the Kerr rotation approaches a constant value less than the saturation value at long time, and this constant value is strongly field dependent. The behavior of the $N=16 \mathrm{sam}$ ple changes from complete reversal at room temperature to less than complete reversal at $96 \mathrm{~K}$, which is consistent with thermal activation processes. These results can be interpreted either as suggesting that defects are more important in the thicker samples or that magnetostatic interactions (due to perhaps subtle domain structures) depend on sample thickness. It is not easy to distinguish between these two mechanisms.

The magnetization reversal process was also investigated by polarized light microscopy. The samples were first saturated magnetically and the Kerr rotation signal was monitored as the field was reversed. When the signal
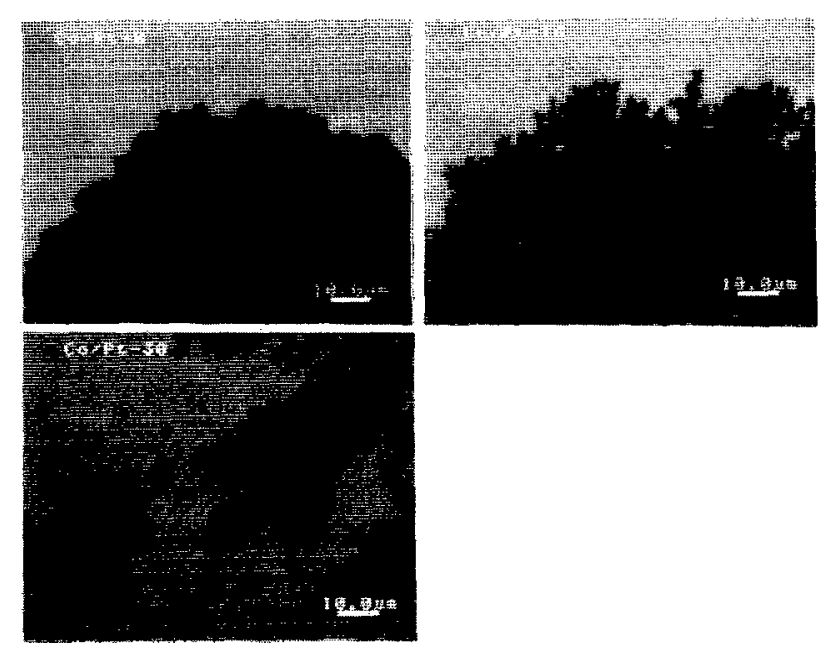

FIG. 3. Domain structure after the Kerr signal changes about $10 \%$ of the peak-to-peak value for $N=8,16$, and 30 samples.

changed by $\sim 10 \%$ of the peak-to-peak value, the sample was removed and placed in the polarizing microscope. Figure 3 shows the resulting domain growth patterns for all three samples. These observations show that domains are nucleated at isolated defect sites or at the edge of the sample. Once the domain is nucleated, it grows rapidly. From Fig. 3, we see that the domain expansion process is very uniform for the $N=8$ sample. There is some raggedness about the edge of the domain, but no unreversed areas are found in the interior region. The expansion for the $N=16$ sample is also quite uniform, but there are some unreversed hard pinning sites in the interior of the domain. The domain shape for the $N=30$ sample is quite irregular, with no evidence of uniform expansion. These results are consistent with the reversal behaviors discussed in reference to Fig. 2, but they suggest that defects rather than magnetostatic effects control the reversal process. However, there is no obvious evidence from the TEM results of Suzuki et al. ${ }^{1}$ that defect structures depend on sample thickness.

To further study the characteristics of defects that pin domain wall motion, we consider a simple uniaxial anisotropy model. Because the applied field is smaller than the coercive field, the movement of the wall is thermally activated. The energy barrier the wall must overcome is given by

$$
E_{W}=E_{U}-H M_{S} V,
$$

where $H, M_{S}$, and $V$ are the applied reversing field, saturation magnetization, and the Barkhausen volume of the irreversible domain wall propagation, respectively, and $E_{U}$ is the activation energy associated with domain wall motion assuming only one $E_{W}$ is present. The time $t_{1 / 2}$ that it takes for the magnetization to decay from the saturated state to the demagnetized state should follow the Arrhenius-Néel law

$$
t_{1 / 2}=t_{0} \exp \left(\frac{E_{W}}{k_{B} T}\right)
$$

so that 


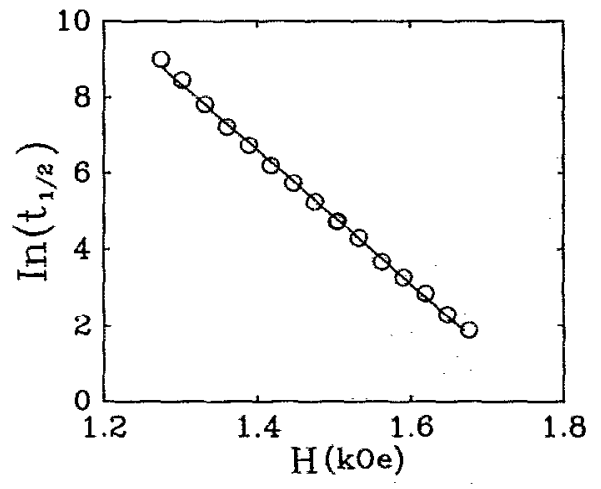

FIG. 4. In $t_{1 / 2}$ as a function of applied reversing field $H$ for $N=8$ sample.

$$
\ln t_{1 / 2}=\ln t_{0}+\frac{E_{U^{-}}-H M_{S} V}{k_{B} T}=A(T)-B(T) H,
$$

where $A(T)=\ln t_{0}+E_{U} / k_{B} T$ and $B(T)=M_{S} V / k_{B} T$. Figure 4 shows one example of the plot of the $\ln t_{1 / 2}$ vs $H$ for the $N=8$ sample. It clearly shows that $\ln t_{1 / 2}$ depends linearly on $H$. Similar behavior is found for the $N=16$ and 30 samples. From this plot and the measured saturation magnetization $M_{S}$, the average volume determined from Eq. (3) can be estimated to be 2.3, 2.6, and $2.9 \times 10^{-18}$ $\mathrm{cm}^{3}$ for $N=8,16$, and 30 , respectively. This is presumably a measure of the volume that reversed by thermal activation during wall motion. The actual volume of defects responsible for wall pinning could be much smaller than this, as suggested by the results of Suzuki et al. ${ }^{1}$ From the decay curves at different temperatures, we can determine $A(T)$ and plot $A(T)$ vs $1 / T$. Figure 5 shows this plot for the $N=8$ and 16 samples. We see that for $N=8$, it is perfectly a straight line, and from the slope of this line we find the activation energy for the domain wall motion to be $E_{U}$ $=1.2 \mathrm{eV}$. This energy barrier may originate from variations in interface roughness that could affect the height of the local anisotropy barrier. For the $N=16$ and $N=30$ samples, there is certainly not a straight line, indicating either a broad distribution of activation energies or a single magnetization-dependent activation energy. This is consistent with the results obtained from the decay measurements for the $N=16$ and 30 samples.

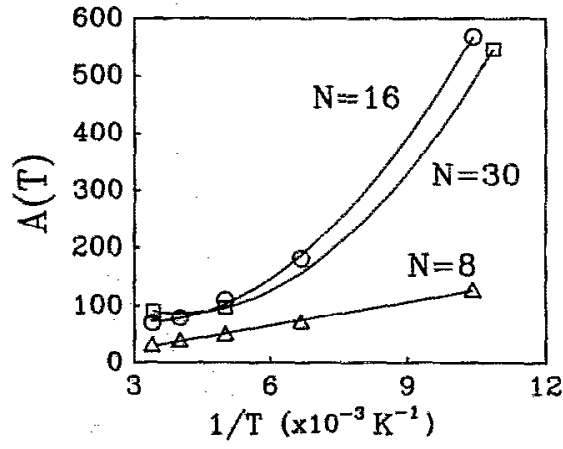

FIG. 5. $A(T)=\ln t_{0}+E_{t^{t}} / k_{B} T$ as a function of $1 / T$ for $N=8$ and 16 samples.

In summary, we have studied the magnetization reversal through the magnetization time dependence and direct domain observations for $\operatorname{Co} 3 \AA / \mathrm{Pt} 10 \AA$ with different numbers of bilayers. We found that magnetization reversal occurs through rare nucleation events following fast domain expansion, and since the intrinsic magnetic properties are the same for all three samples it seems likely that the expansion process is controlled by presence of defects. These defects may originate from the roughness of the interface between the Co and $\mathrm{Pt}$, as suggested by Suzuki et $a l^{1}{ }^{1}$ When the films become thicker, our results indicate a change in the character of the energy barriers involved in wall motion. These changes appear to involve defects that lead to a distribution of barrier heights in thicker samples.

We gratefully acknowledge financial support from the Research Corporation and from the NSF under Grant DMR-8918889.

${ }^{1}$ T. Suzuki, H. Notarys, D. C. Dobbertin, C.-J, Lin, D. Well, D. C. Miller, and G. Gorman, IEEE Trans. Magn. 28, 2754 (1992).

${ }^{2}$ M. Labrune, S. Andrieu, F. Rio, and P. Bernstein, J. Magn. Magn. Mater. 80, 211 (1989).

${ }^{3}$ J. Pommier, P. Meyer, G. Penissard, J. Ferre, P. Bruno, and D. Renard, Phys. Rev. Lett، 65, 2054 (1990).

${ }^{4}$ R. D. Kirby, J. X. Shen, Z. S. Shan, and D. J. Sellmyer, J. Appl. Phys. $70,6200(1991)$

SJ. X. Shen, R. D. Kirby, and D. J. Sellmyer, J. Magn. Magn. Mater. 81, 107 (1989) 